\title{
Will nursing shortages threaten the integrity and functioning of the NHS?
}

\author{
Emeritus Professor Alan Glasper, from the University of Southampton, discusses a recent Government \\ report that reveals that the current nurse vacancy rate has exceeded NICE's recommended guidelines
}

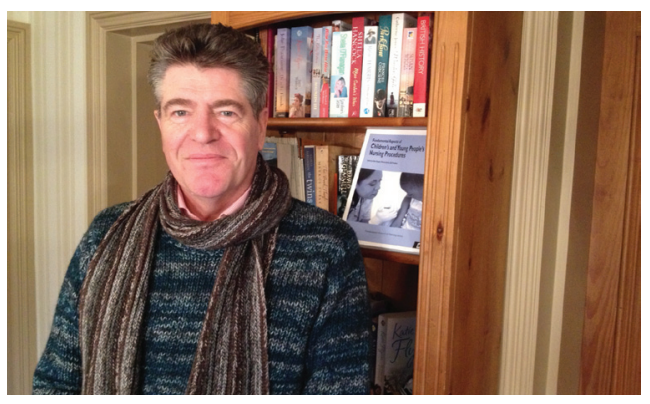

$\mathrm{n}$ the last issue of the $B J N$, the link between patient safety and nurse staffing levels was highlighted (Glasper, 2016).

Healthcare staffing is currently under the spotlight, with the media reporting that the NHS has 70000 fewer staff than the Government previously thought. According to The Guardian, this represents, among others, 15000 fewer nurses and 3000 fewer doctors (Campbell, 2016).

However, the Government has recognised the looming crisis of nurse staffing in the NHS .In October 2015 it commissioned the Migration Advisory Committee (MAC), a non-departmental public organisation linked to the Home Office whose role it is to advise the Government on migration issues and review whether there is a shortage of nurses or specific nursing roles that could filled by non-European Economic Area (EEA) migration. Nurses were subsequently placed on the Shortage Occupation List (SOL) (Gov. uk, 2015) on a temporary basis, pending MAC's review of the evidence (SOL is a list of positions where there are not enough resident workers to fill the available jobs in that particular sector). In March 2016, MAC published its report, entitled Migration Advisory Committee (MAC) partial review: shortage occupation list and nursing.

\section{Background}

The MAC report investigated how many registered nurses are presently employed in the UK. They estimate there are about
630000 qualified nurses working in the UK, of which some 500000 currently work in the NHS. In 2011, the Organisation for Economic Cooperation and Development (OECD) estimated that at least $20 \%$ of these were nurses from overseas. On this basis, MAC believes that there are currently about 140000 foreign-born qualified nurses registered with the Nursing and Midwifery Council (NMC) to practise in the UK. MAC collected data from 106 out of 166 trusts and health boards in England, Wales and Northern Ireland. The data revealed that:

- Between 2013 and 2015, there has been a $50 \%$ increase in nursing vacancies, from 12513 to 18714 .

- In England and Wales, there were 1265 vacancies for registered nurses in emergency departments, representing about $11 \%$ of the total nursing workforce.

Over the past quarter-century, immigration of foreign-born nurses to the UK has at certain periods been a significant answer to nursing shortages and to the overall nursing supply. Yearly inflows to the UK of such nurses peaked at just over 16000 in 2001/02, having been below 5000 a year during most of the 1990s. Nurse inflows remained above 10000 each year up to and including 2005/06, before falling to over 2000 in 2009/10. The number of nurses seeking work in the UK has again risen to around 8000 in 2014/15, with the vast majority arriving from the EEA. Despite this inflow, there was a higher number of vacancies in England than recommended in the National Institute for Health and Care Excellence (NICE) guidelines (NICE, 2014).

MAC has indicated that many senior officers within the health sector continue to see the immigration of nurses as a 'Get-outof-jail-free card' and an answer to the national shortage. Despite government pledges to train more nurses, MAC believes that the current shortage is caused by factors that could, and should, have been anticipated. The MAC publication reports on data it gathered from the Royal College of Nursing (RCN, 2014), which shows that NHS trusts in London have an average nurse vacancy rate of $17 \%$, with some as high as 30\%. It's the London and the south-east trusts that accounted for around two-thirds or more of all nurse certificates of sponsorship used since 2013. Once accepted as sponsors, employers such as hospitals can issue 'Certificates of Sponsorship' to migrant workers such as nurses. Both Scotland and Northern Ireland have lower vacancy rates of only 4\%; Wales's is slightly higher. The RCN has serious concerns that there is a shortage of nurses in the UK and that healthcare providers are struggling to recruit them. In particular, the $\mathrm{RCN}$ is anxious that certain specialty grades such as neonatal nurses and paediatric intensive care nurses are in short supply.

\section{Why are there not enough nurses?}

There is no doubt that demographic change in the patient population-such as an increase in the numbers of elderly people, a crisis in out-of-hours primary health care and the demise of services such as the nurse-led NHS Direct telephone triage provision-is putting an added burden on NHS hospital services, but especially emergency departments. The role of the nurse has also changed, which some attribute to the introduction of the European Working Time Directive (EWTD) for junior doctors. Limiting working hours can help reduce the likelihood of doctors getting tired, and the EWTD was introduced with the aim of improving the quality of service junior doctors give to patients (British Medical Association (BMA), 2016).

However, in some cases, the EWTD led to gaps in junior doctor rotas and new nurseled services were introduced to address them. For example, Campbell and Spencer (2007) highlight the evolution of new nursing roles within the specialty of neonatal care, where shortages of suitably qualified nurses are giving 
rise to concern about the ability of neonatal units to meet British Association of Perinatal Medicine (BAPM) standards, which in neonatal intensive care demands one neonatal nurse to one baby. The introduction of roles such as advanced neonatal nurse practitioner has been designed to address skill-mix deficits and to ease the medical workforce challenges caused by the EWTD. However, as nurses have embraced roles previously occupied by junior doctors, in turn many former nursing roles have been filled by healthcare assistants (HCAs). Workforce and role changes have also been exacerbated by changes to safe-staffing guidelines in the wake of public inquires such as the Francis report (2013) into the Mid Staffordshire NHS Foundation Trust.

In 2016, regulators such as the Care Quality Commission (CQC) are inspecting off-duty rotas to ensure that safe staffing levels are actually achieved across the full working week - because research has linked adverse patient outcomes to nurse staffing ratios. For example, in a cross-sectional analysis of survey data, Rafferty et al (2007) showed that hospitals with optimal staffing levels had consistently better outcomes than those with less favourable staffing. The MAC report also cites evidence from nurses that working conditions - such as stress, burnout, lack of job satisfaction and work environment-were prime reasons for leaving the profession. In addition, a high proportion of registered nurses are nearing retirement age and many are taking the opportunity to leave.

One of the more contentious issues raised by the MAC report is the number of nurse training paces available in the country and the failure to educate sufficient numbers of future nurses. At least part of the current shortage has been attributed to the decision to cut training places in England by almost a fifth between 2009 and 2013. Some of the evidence MAC received indicated that the numbers of nurse training commissions funded in recent years has been more influenced by financial pressures than by anticipated clinical demand. Worryingly, MAC has also ascertained that, on average, migrant nurses are being paid $£ 6000$ less than equivalent UK nurses, and that some employers might be using non-EEA nurses for $\Xi$ fiscal reasons rather than properly addressing the shortage through other mechanisms. Matters have been exacerbated by poor workforce planning which, until recently, did not take into account the demand for nurses in the care and independent sectors of society.
Undoubtedly, the ongoing financial pressures on NHS finances may continue to motivate managers to recruit migrant nurses at lower cost for the sake of their budgets.

\section{MAC recommendations}

MAC has reservations about keeping nursing on the SOL because doing so might lead to nurses procuring a disproportionate number of the certificates of sponsorship available to skilled workers under the Tier 2 limit, to the detriment of other employers. The Government has made it clear that it is imposing an overall cap of 20700 persons per year. To avoid confrontation with other employment sectors, MAC is suggesting a yearly cap of 5000 places for nurses under Tier 2 , with the limit falling gradually over the next 3 years. (Note: for an employment position such as nursing to be added to the Tier 2 SOL, it must be skilled to National Qualifications Framework (NQF) Level 6, the equivalent of a university degree).

MAC is also suggesting that employers wishing to recruit non-EEA nurses should be required to complete a resident labour market test to allay fears that NHS or other health managers will recruit nurses from outside the EEA simply to avoid paying standard UK nursing rates. In 2014-15, 8000 foreign-born nurses were recruited, mainly from the EEA. It is important to stress that the NMC has already developed rigorous processes for assessing the suitability of non-EEA overseas applicants to join the register (Glasper, 2013; 2014).

The chair of MAC has emphasised in the report that the country should be able to maintain its own supply of nurses without having to rely on overseas or EEA recruitment. Cuts in the commissions of undergraduate student nurse places at universities might be mitigated by the replacement of bursaries in favour of student loans. Chancellor George Osbourne believes that replacing bursaries with loans will give more people the opportunity to study. However, many nursing academics are fearful that tuition fees of $£, 9000$ per year may force some applicants to seek access to nurse training through the recently announced apprenticeship schemes, leading to nursing-associate status where the brunt of fees can be underwritten by employers. Nursing associates will be able to access further training, either through full degree-level nurse apprenticeships or through shortened nursing degree courses at university (Department of Health (DH), 2015).

\section{Conclusion}

The MAC report concludes that there is currently a shortage of nurses in the UK, with much of this shortage being most significant in England. The committee's remit was to consider whether there is a UK-wide shortage; they believe that there is, and have therefore recommended that nursing be retained on the SOL. However, the longterm solution to addressing this shortage is the training, recruitment and retention of nurses by providing sufficient incentives and opportunities for them in the NHS. BJN

British Medical Association (2016) European Working Time Directive. http://tinyurl.com/gn2k2yt (accessed 15 April 2016)

Campbell C, Spencer SA (2007) The implications of the Working Time Directive: how can paediatrics survive? Arch Dis Child 92(7): 573-5

Campbell D (2016) NHS has 70,000 fewer staff, new figures reveal. Guardian online. Sunday 3 April 2016

Department of Health (2015) Nursing associate role offers new route into nursing. http://tinyurl.com/hwgsbfe (accessed 15 April 2016)

Francis R (2013) The Mid Staffordshire NHS Foundation Trust Public Inquiry. http://tinyurl.com/obgqflx (accessed 15 April 2016)

Glasper A (2013) NMC reviews registration of nurses trained outside the EU. Br J Nurs 22(10): 586-7

Glasper A (2014) New registration process for overseas nurses. Br J Nurs 23(15): 862-3

Glasper A (2016) Moving from a blame culture to a learning culture in the NHS. Br J Nurs 25(7): 342-3

Migration Advisory Committee (2016) MAC partial review: shortage occupation list and nursing. http://tinyurl.com/ hv38jt2 (accessed 15 April 2016)

National Institute for Health and Care Excellence (2014) Safe staffing for nursing in adult inpatient wards in acute hospitals. SG1. www.nice.org.uk/guidance/sg1 (accessed 15 April 2016)

Rafferty AM, Clarke SP, Coles J, et al (2007) Outcomes of variation in hospital nurse staffing in English hospitals: cross-sectional analysis of survey data and discharge records. Int J Nurs Stud 44(2):175-82

Royal College of Nursing (2014) Response to Migration Advisory Committee call for evidence: Partial review of the shortage occupation lists for the UK and Scotland. http://tinyurl.com/zmlkvzr (accessed 15 April 2016)

\section{KEY POINTS}

Data from the Migration Advisory Committee has shown that the NICE recommendation for nurse staffing are not being met by many NHS institutions

- NHS Trusts in London have an average nurse vacancy rate of $17 \%$, with some as high as $30 \%$

- Shortfalls in nursing numbers have been have been caused by reductions in the number of training places available and a failure to educate enough nurses to enter the profession

- Nurses have been placed on the UK Shortage Occupation List (SOL) 